\title{
Assessment of Active and Passive Restraint During Guided Reaching After Chronic Brain Injury
}

\author{
DA VID J. REINKENSMEYER, ${ }^{\mathbf{l}, 2}$ BRIAN D. SCHMIT, ${ }^{2}$ and WILLIAM Z. RYMER ${ }^{2}$ \\ 'Department of Mechanical and Aerospace Engineering, and Program in Biomedical Engineering, University of California, Irvine, \\ CA and ${ }^{2}$ Department of Physical Medicine and Rehabilitation, Northwestern University Medical School, and Sensory Motor \\ Performance Program, Rehabilitation Institute of Chicago, Chicago, IL
}

(Received 9 April 1999; accepted 31 August 1999)

\begin{abstract}
We report the use of a mechatronic device for assessing arm movement impairment after chronic brain injury. The device, called the "Assisted Rehabilitation and Measurement Guide," is designed to guide reaching movements across the workspace, to measure movement and force generation, and to apply controlled forces to the arm along linear reaching paths. We performed a series of experiments using the device in order to identify the contribution of active muscle and passive tissue restraint to decreased active range of motion of guided reaching [i.e., "workspace deficits"' in a group of five chronic, spastic hemiparetic, brain-injured subjects. Our findings were that passive tissue restraint was increased in the spastic arms, as compared to the contralateral, nonparetic arms. Active muscle restraint, on the other hand, was typically comparable in the two arms, as quantified by measurements of active arm stiffness at the workspace boundary during reaching. In all subjects, there was evidence of movement-generated weakness, consistent with a small contribution of spasticity to workspace deficits. These results demonstrate the feasibility of mechatronic assessment of the causes of decreased functional movement, and could provide a basis for enhanced treatment planning and monitoring following brain injury. (C) 1999 Biomedical Engineering Society. [50090-6964,99,01106-6]
\end{abstract}

Keywords-Rehabilitation, Neurological control systems, Bioinstrumentation, Biomechanics, Spasticity

\section{INTRODUCTION}

Recently there has been a surge of interest in bringing mechatronic and robotic technology to bear on rehabilitation of movement after brain injury. ${ }^{26,27}$ Key factors motivating this interest are a need for quantitative evaluation in rehabilitation, and the potential to provide increased therapy "dosage" at reduced cost through automation of amenable manual therapy techniques. Stroke is currently a leading cause of severe disability in the U.S., and arm and hand movements are often impaired after stroke. A significant amount of recent research has therefore been focused on devices for assessment and therapy of the arm after stroke. ${ }^{\mathbf{l} 21,30}$ Such devices could ulti-

Address correspondence to David J. Reinkensmeyer. Electronic mail: dreinken@uci.edu mately benefit approximately 300,000 new stroke survivors per year, as well as the more than 1.5 million chronic stroke survivors with movement disability in the U.S. ${ }^{17}$

One of the ways that mechatronic devices could impact neurorehabilitation is to improve quantitative assessment of arm movement. Brain injury ‘including stroke' is often accompanied by a series of motor impairments, including weakness, reduced passive tissue compliance, spasticity ґdefined as hyperactive stretch reflexes', and impaired motor coordination. The severity of these impairments may vary from patient to patient. Objective assessment of the relative contributions of these different motor impairments to reduced functional movement ability would improve treatment planning.

In a pilot study we used a mechatronic device to evaluate reaching capability following brain injury. ${ }^{30}$ The device, called the "Assisted Rehabilitation and Measurement Guide' ‘ARM Guide', consists of a linear constraint that guides reaching across the subject's workspace, measuring range of motion and contact force generation [Fig. 1'. We found that brain-injured subjects exhibited highly repeatable active range of motion deficits during reaching along the ARM Guide. In other words, the usable "workspace" of the arm, defined as the volume of space that the hand could reach voluntarily, was reproducibly reduced on sequential movement attempts. Workspace deficits were consistent even though the subjects exhibited grossly abnormal coordination during reaching, manifested as large contact forces against the ARM Guide.

The goal of the present study was to identify to what extent increased passive tissue and active muscle restraint contribute to such workspace reductions. Increased passive tissue restraint may arise following brain injury from shortening and stiffening of muscle and connective tissue, due to disuse and persistent abnormal posture of the spastic arm. ${ }^{15,24}$ Active muscle restraint may arise from uncontrolled stretch reflex activation of antagonists during reaching 『i.e., spasticity ${ }^{20,25}$, or from 


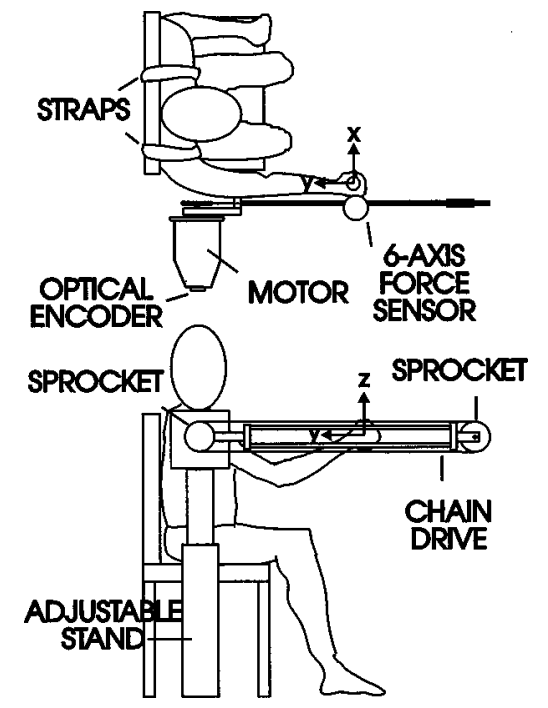

FIGURE 1. Experimental apparatus (the "ARM Guide"). The subject's forearm/hand was attached to a handle that slid along a linear constraint via a low-friction, linear bearing. A six-axis force/torque sensor sensed contact forces between the hand and the constraint in the coordinate system shown. A computer-controlled motor attached to a zero-backlash chain drive was used to drive the hand along the constraint. An optical encoder measured the distance traveled by the hand along the constraint.

abnormal antagonist coactivation characterized by involuntary spreading of voluntary activation commands. ${ }^{8,18}$ Either restraint mechanism would cause an increased resisting force to voluntary movement of the arm, potentially contributing to the establishment of workspace deficits.

To assess levels of restraint during reaching, we performed a series of experiments with the ARM Guide on five chronic, brain injured subjects with clinically identified spasticity at the elbow. To quantify passive tissue restraint, we used the ARM Guide to measure the resisting force generated by the subject's relaxed arm at the workspace boundary. To quantify active muscle restraint, we measured the active stiffness of the arm at the workspace boundary, immediately following reaching to the boundary while the subject was still activating muscles and trying to reach farther. Since stiffness has been found to be proportional to force generation in an isolated muscle ${ }^{23}$ as well as at the elbow and shoulder, 3,16 the measurements of active stiffness were taken to reflect the force generation in antagonist muscles, i.e., active restraint. We also performed an experiment to determine whether "movement-generated weakness," defined as a loss of force generating ability due to a preceding voluntary movement, contributes to weakness at the workspace boundary. Movement-generated weakness might arise, for example, if spastic reflexes in antagonist muscles are triggered by reaching and persist at the workspace boundary.
Our findings were that passive tissue restraint was typically increased at both the shoulder and elbow in the spastic arms, as compared to the contralateral, nonparetic arms. On the other hand, active muscle restraint was comparable in the two arms. In all subjects, there was evidence of movement-generated weakness. Portions of this work have been reported in abstract form. ${ }^{29}$

\section{METHODS}

\section{Subjects}

Five subjects were selected for inclusion in the study according to the following criteria: each sustained a hemispheric stroke or traumatic brain injury at least six months previously; unilateral paresis arising from the brain injury; spasticity in elbow flexor muscles as detected using passive manual stretches of the elbow; no evidence of significant subluxation of the shoulder or shoulder pain; absence of cognitive dysfunction or aphasia sufficient to limit comprehension of the experimental task; no neglect or apraxia; no concurrent severe medical problems.

The clinical features of the subjects are summarized in Table 1. All subjects had spasticity present in the elbow flexors classifiable as mild or moderate according to the Ashworth scale. ${ }^{2}$ The spasticity of the braininjured subject was clinically indistinguishable from that of the stroke subjects. All subjects also exhibited significant loss of arm movement ability as graded using the Fugl-Meyer Motor Performance Scale. ${ }^{\mathbf{1 3}}$ None of the subjects had evidence of spasticity, abnormal synergetic movement, or decreased passive range of motion of the contralateral arm, and all of the subjects used their contralateral arm regularly to perform activities of daily living. Thus, in this study the contralateral arm was used to establish subject-specific, normative reaching behavior, although it is recognized that the motor capacity of this arm may also have been subtly affected by the hemispheric brain injury. ${ }^{7}$

All subjects provided informed consent, the Institutional Review Board of Northwestern University approved all experiments, and all procedures were conducted in conformity with the Declaration of Helsinki.

\section{Description of ARM Guide}

Subjects reached along a linear constraint provided by a custom-built mechanical device, the "ARM Guide", 30 'Fig. 1'. Briefly, the device is a linear constraint that is instrumented with a six-axis load cell 'ATI Industrial Automation's and an optical encoder. Further, a computer-controlled torque motor 'PMI Inc.'’ is incorporated into the device in order to apply forces or controlled movements to the arm along the linear constraint. The device is capable of being oriented at different di- 
TABLE 1. Clinical features of subjects, and movement parameters measured during reaching with the spastic arm along the ARM Guide.

\begin{tabular}{ccccccrrrrr}
\hline Subject & Age & Sex & Etiology & $\begin{array}{c}\text { Paretic } \\
\text { side }\end{array}$ & $\begin{array}{c}\text { Duration } \\
\text { (years) }\end{array}$ & $\begin{array}{c}\text { Ashworth } \\
\text { score }\end{array}$ & $\begin{array}{c}\text { Fugl-Meyer } \\
\text { score }\end{array}$ & $\begin{array}{c}\text { Movement } \\
\text { amplitude [cm] } \\
\text { mean (SD) }\end{array}$ & $\begin{array}{c}\text { Remaining distance to } \\
\text { passive mechanical } \\
\text { Limit [cm] }\end{array}$ & $\begin{array}{c}\text { Peak velocity } \\
\text { [cm/s] } \\
\text { mean (SD) }\end{array}$ \\
\hline A & 49 & M & TBI & L & 18 & 2 & 12 & $10.0(1.0)$ & 14.5 & $46.9(8.3)$ \\
B & 52 & M & CVA & R & 3 & 2 & 17 & $7.6(0.7)$ & 13.8 & $42.8(6.8)$ \\
C & 63 & F & CVA & R & 4 & 3 & 24 & $10.3(1.4)$ & 9.3 & $24.1(5.2)$ \\
D & 55 & F & CVA & R & 4 & 2 & 39 & $16.7(1.5)$ & 6.9 & $43.4(6.5)$ \\
E & 74 & M & CVA & L & 3 & 1 & 27 & $15.1(0.6)$ & 16.0 & $71.0(2.7)$ \\
\hline
\end{tabular}

${ }^{a}$ TBI: traumatic brain injury (focal type): CVA: cerebrovascular accident (all ischemic type).

${ }^{\mathrm{b}}$ Ashworth Scale of Tone ${ }^{\mathbf{2}} \mathbf{0} \mathbf{0}=$ normal tone; $1=\mathbf{s i g h t}$ increase in tone, giving "catch" when the limb is moved; 2 =more marked increased in tone, but limb easily moved; $3=$ eonsiderable increase in tone, passive movement difficult; 4 =limb rigid in flexion or extension.

${ }^{c}$ Fugl-Meyer Score (Ref. 13) is out of a possible 66 for the upper extremity.

${ }^{d}$ Movement amplitude is the final resting position of hand during reaching along the ARM Guide, relative to the starting hand position. ${ }^{e}$ Remaining distance to passive mechanical limit is the mean distance following active reach to a safety stop set $5 \mathrm{~cm}$ before the full passive range of motion of the subject, as determined by the experimenter by manually extending the subject's passive arm along the ARM Guide.

${ }^{\mathrm{f}}$ Peak velocity is the peak linear hand velocity achieved during reaching along the ARM Guide. SD=standard deviation.

rections in the vertical plane. For the present study, the device was always oriented so that the linear constraint was horizontal 'Fig. 1 '’.

To use the ARM Guide, the subject's hand was wrapped around a wooden cone, and the forearm was attached to a specially designed splint using straps. The splint slid along the linear constraint using a low-friction, linear ball bearing. The splint also prevented movement at the wrist joint, and did not require the subject to have the ability to grasp. To apply position or force perturbations to the arm, the motor drove a chain drive attached to the splint. Measurement of hand position was achieved by an optical encoder attached to the motor. Measurement of forces generated at the hand was provided by the six-axis load cell mounted between the splint and linear constraint.

For the experiments in this paper, the encoder and load cell signals were sampled and the motor was controlled at $200 \mathrm{~Hz}$ using dedicated data acquistion and control cards on a PC. To apply stretches to the arm along the ARM Guide, the motor was controlled with a high gain proportional/derivative positional feedback controller implemented in the $\mathrm{C}$ programming language. The controller achieved tracking errors of less than 1.5 $\mathrm{mm}$ across a range of speeds. The desired trajectory input to the controller for the stretches depended on the particular experimental condition, as explained in the next section.

\section{Experimental Protocol}

Subjects sat with their torso securely strapped to a chair, using straps positioned around the chest and across the clavicles, to prevent trunk movement. The height and position of the ARM Guide were adjusted such that the rotation axis of the motor was aligned with the approximate rotation axis of the shoulder in flexion/extension. Alignment was assured using anatomical landmarks, determined by palpating the bony head of the humerus. Arm abduction was set between $10^{\circ}$ and $20^{\circ}$, so that movements along the ARM Guide primarily required shoulder and elbow flexion and extension.

For safety, a mechanical stop was set at the end of the ARM Guide. The stop prevented the subject's arm from extending through its full passive range of motion. The experimenter determined the position of the stop by manually extending the subject's arm along the ARM Guide. The stop was set so that the arm could not move closer than approximately $5 \mathrm{~cm}$ to its full passive mechanical limit.

Subjects began each experiment by practicing reaching along the device 12 times with the spastic arm. The arm was initially placed in an elbow-flexed position ‘ $60^{\circ}-84^{\circ}$ angle between upper arm and forearm' at which it remained stationary without voluntary intervention by the subject. Extending the arm outward from this position required that the subjects overcome not only restraint forces generated by passive tissues and antagonist muscle activity, but also gravitational forces. A simple way to understand these gravitational forces is that the center of mass of the arm was at a lower height in the elbow-flexed position than with the arm fully extended along the ARM Guide. Thus, gravitational forces acted to lower the center of mass toward the flexed position, moving the passive arm along the ARM Guide even though the constraint was in a horizontal orientation.

To perform reaches, subjects were instructed to "move as far and as fast as possible, and to try not to 
TABLE 2. Summary of experimental protocol.

\begin{tabular}{|c|c|}
\hline Practice reaches ${ }^{a}$ & Reaches interspersed with mechanical tests ${ }^{\mathbf{b}}$ \\
\hline $\boldsymbol{A}_{1} \boldsymbol{A}_{2} \ldots \boldsymbol{A}_{12} 5$ min rest & $\boldsymbol{A}_{1(T S)} \boldsymbol{P}_{1(T S \boldsymbol{M}} \boldsymbol{M G W} \mathbf{A}_{1} \boldsymbol{A}_{2(T S)} \boldsymbol{M G W} W_{2} \boldsymbol{P}_{2(T S)} \ldots \boldsymbol{A}_{12(T S)} \boldsymbol{M} G W_{12}$ \\
\hline $\begin{array}{l}A_{\mathbf{I}}: \text { Active reach. } \\
A_{\mathbf{I}(\boldsymbol{T} S)}: \text { Active restraint } \\
\text { active arm stiffness. } P_{\mathbf{1}} \\
\text { active range achieved b } \\
\text { stiffness. } M G W_{1}: \text { Move } \\
\text { arm was returned to a } \\
\text { reach. Subject rested a } \\
\boldsymbol{P}_{\mathbf{I}(\boldsymbol{T}) \text { ) was randomized }}\end{array}$ & $\begin{array}{l}\text { ach followed immediately by } 4 \mathrm{~cm} \text { terminal stretch to evaluat } \\
\text { restraint test-slow passive movement of the arm through th } \\
\text { ated weakness test-performed only on spastic arms. Subject' } \\
\text { ositioned at the workspace boundary achieved by the previou } \\
\text { then attempted to reach again. Relative order of } M G W_{\mathbf{I}} \text { an } \\
(\boldsymbol{T} S) \text {. }\end{array}$ \\
\hline
\end{tabular}

push up or down, or to the left or right against the device." Reaches were initiated at the verbal cue of the experimenter. Subjects were also verbally informed of the numerical value of the final endpoint position after each reach "in centimeters, referenced from the starting hand position', and also visually monitored their arm. After each reach, the motor was used to move the arm slowly back to the position at which the reach began. Subjects were given 5-10 s of rest between each movement condition to minimize muscular fatigue.

Two subjects 'subjects D and E' had full active range of motion with the spastic arm during supported movement along the ARM Guide, even though they did not have full active range of motion during unsupported reaching. To induce a workspace deficit in these subjects along the ARM Guide, the motor was used to increase the loading on the arm. A linear spring load with a stiffness of $2.5 \mathrm{~N} / \mathrm{cm}$ was applied, and the resting length of the spring load was set to be at the initial resting position of the hand, so that the subjects were not required to support the load prior to reaching. For these two subjects, a matched spring load was also applied to the contralateral arm during reaching.

After the subjects performed an initial 12 practice reaches exhibiting workspace deficits, they rested for 5 mins. They then performed a second set of 12 reaches that were interspersed with three mechanical tests isummarized in Table 2'j. These tests were the passive restraint test, active restraint test, and movement-generated weakness test, as described in detail next.

Passive Restraint Test. To evaluate the level of passive tissue restraint at the workspace boundary, the ARM Guide returned the subject's hand to the position from which the most recent reach was initiated. Then, while the subject relaxed the arm, the device moved the arm slowly back to the workspace boundary achieved by the most recent reach, and the force needed to hold the passive arm at the boundary was measured 'Fig. 2 top'. The slow passive movement followed a fifth-order polynomial position profile, with a peak velocity of $4.0 \mathrm{~cm} / \mathrm{s}$, and amplitude equal to that of the previous voluntary reach. Thus, at the end of the passive movement, the subject's hand was at the workspace boundary defined by the previous reach 'Fig. 2 top'. During these slow passive movements, EMG recordings of seven muscles sur-
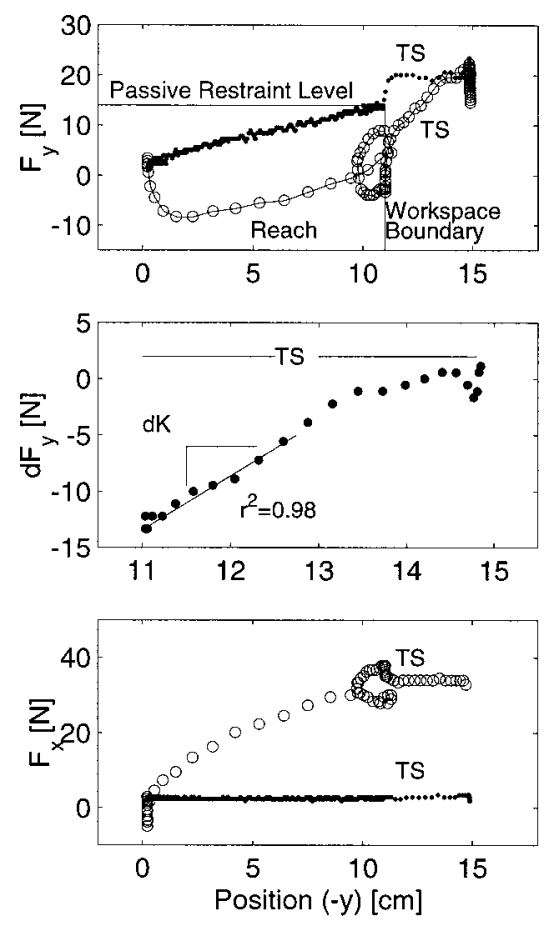

FIGURE 2. Top: Force measured in the direction opposite movement along the ARM Guide (i.e., in the $y$ direction in Fig. 1) for the passive restraint (dots) and active reach [ circles] conditions for spastic arm of subject B. At the end of each movement condition ¿i.e., at the workspace boundary!, a terminal stretch [TS was applied to the arm. Time between points is 20 ms. Middle: By subtracting the forces measured during the terminal stretches for each movement condition, an approximately linear relationship between differential force $\left[d F_{,}\right\}$and arm position was obtained. A linear regression was performed over the first $200 \mathrm{~ms}$ of the response to identify its slope $\{d \mid \backslash \backslash$, the active arm stiffness. Time between points is again $20 \mathrm{~ms}$ for graphical clarity, although samples taken every $5 \mathrm{~ms}$ were used in the regression. Bottom: Off-axis force measured in the $x$ direction i Fig. 1) during reaching for the passive restraint [dotsi and active reach ' circles' conditions. 
rounding the shoulder and elbow were used to verify that muscles were inactive [see below'. For comparison, the passive force generated by the contralateral arm at a matched position was also evaluated.

Active Restraint Test. We hypothesized that any active restraint arising from activation of antagonist muscles during reaching would manifest itself as an increased stiffness following reaching, while the subject was still activating muscles and trying to move beyond the workspace boundary. To evaluate this stiffness, a small stretch Ithe "terminal stretch', ' of $4 \mathrm{~cm}$ amplitude was applied to the arm when hand velocity had dropped and remained below $1 \mathrm{~mm} / \mathrm{s}$ for $150 \mathrm{~ms}$. The terminal stretch duration was $500 \mathrm{~ms}$, and the stretch profile was chosen to be a fifth-order polynomial 'bell-shaped velocity profile, peak velocity $15.0 \mathrm{~cm} / \mathrm{s}$ in order to minimize inertial transients due to the acceleration of the mass of the arm. The fifth-order polynomial profile was used as the desired trajectory to the feedback controller described above. An identical small stretch was applied following the slow passive movement of the arm through the same range for comparison [Fig. 2 top'.

Movement-Generated Weakness Test. A third test was performed to identify whether the preceding voluntary movement of the arm contributed to weakness at the workspace boundary, as would be expected, for example, if spastic reflexes are triggered by reaching and persist at the workspace boundary. For this test, the ARM Guide moved the subject's relaxed arm back to the workspace boundary achieved during the most recent reach. A virtual "wall" was then created using the motor to support the hand at the workspace boundary. The wall prevented movement of the subject's hand back toward the shoulder, but allowed movement away from the shoulder. After resting for $10 \mathrm{~s}$ against the virtual wall lifuring which time EMG recordings were used to confirm that arm muscles were inactive', the subject attempted to reach farther along the ARM Guide at the experimenter's verbal cue. This test and the passive restraint test were applied in random order following each reach, to eliminate possible order effects ‘Table 2'j.

Reaching movements were also recorded from each subject's contralateral arm. These movements were matched to the movements of the spastic arm, in terms of initial starting position, amplitude, and peak velocity. Subjects were instructed to move smoothly, and to match the position of a marker on the hand splint to a $2.0-\mathrm{cm}-$ wide visual target placed beside the linear shaft of the ARM Guide. After each reach, subjects were verbally informed of the actual and desired peak velocity of movement, as determined by digitally differentiating the reach profile. Subjects practiced reaching with the contralateral arm twelve times in order to become proficient in achieving the desired amplitude and velocity. They then performed twelve reaches, with each reach followed by a terminal stiffness test. Also, as with the spastic arm, the terminal stiffness test was followed by a passive restraint test. The movement-generated weakness test was not repeated for the contralateral arm because all subjects were capable of extending the contralateral arm to its full mechanical limit.

\section{Electromyography}

Surface EMGs were recorded from seven superficial shoulder and elbow muscles using disposable silver/ silver chloride hydrogel electrodes [Electrotrace ET 301. The primary purpose of these EMG recordings was to confirm that muscles were inactive during the passive restraint test and before the movement-generated restraint test, but the recordings also provided some indication of muscle activation patterns during reaching. EMG recordings were obtained from the brachioradialis, biceps, lateral head of triceps, horizontal fibers of pectorialis major, and the anterior, intermediate, and posterior deltoid muscles. Electrode placements followed the general guidelines found in Delagi and Perotto. ${ }^{6}$ The skin was cleaned with alcohol and lightly abraded before placing the electrodes. The ground lead was placed on the bony surface of the elbow.

EMG signals were differentially amplified with a gain of 10, then high-pass filtered with a two-pole Butterworth filter with a cutoff of $6 \mathrm{~Hz}$. They were then amplified by a factor of 2000 and low passed filtered using an eight-pole Butterworth filter with a cutoff of $250 \mathrm{~Hz}$. The filtered signals were sampled at $500 \mathrm{~Hz}$ using an analog-to-digital 'A/D' board with custom LabVIEW software and stored on a PC. For graphical presentations shown below, EMGs were digitally rectified and smoothed using a 20 point, causal moving average filter.

\section{Data Analysis}

To quantify passive restraint, the resisting force in the $y$ direction 'Fig. 1' generated by the spastic arm at the end of the slow passive stretch was averaged across stretches. For comparison, the resisting force generated by the contralateral arm following the matched slow stretch was averaged. In addition, using a simple twojoint biomechanical model of the arm, the individual contributions of shoulder flexion/extension and elbow flexion/extension torques to the resisting force at the hand were calculated. In particular, the joint torques were related to the $y$ and $z$ components 'Fig. 1' of the hand force by static equations for a two-revolute joint arm: ${ }^{5}$ 


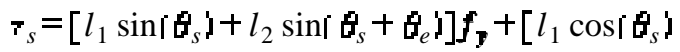

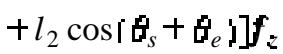

$$
\begin{aligned}
& \tau_{e}=l_{2}\left[\operatorname { s i n } \left\lceil\theta_{s}+B_{e} d f_{y}+\cos \left[B_{s}+B_{e} d f_{g}\right]\right.\right.
\end{aligned}
$$

where $\boldsymbol{\tau}_{s}$ and $\boldsymbol{\tau}_{\boldsymbol{e}}$ are the shoulder flexion/extension and elbow flexion/extension torques defined positive for flexion; $\boldsymbol{I}_{1}$ and $\boldsymbol{I}_{2}$ are the lengths of the lower and upper arms; and $\boldsymbol{t}_{s}$ and $\boldsymbol{\theta}_{e}$ are the shoulder flexion and elbow flexion angles, defined as zero when the arm is fully outstretched along the ARM Guide, and increasing with shoulder flexion and elbow flexion, respectively. The forces $f_{y}$ and $f_{z}$ are the forces measured at the hand by the load cell following the slow-passive stretch, with $f_{y}$ being the force parallel to the ARM Guide idlefined positive in the direction resisting stretchj; and $\boldsymbol{f}_{z}$ the force perpendicular to the ARM Guide in the vertical plane 'defined positive upward' 'see Fig. 1'j. These equations do not include dynamic terms li.e., inertial, centripetal, and coriolis forces' because the restraint force was measured under static conditions li.e., the arm had ceased moving'. The shoulder and elbow flexion angles were calculated using the standard two-revolute joint arm inverse kinematic equations. ${ }^{5}$

To evaluate the active stiffness of the arm following reaching, the following procedure was adopted. The force response to the terminal stretch following slow passive movement was subtracted from that following reaching, and the differential force was plotted as a function of position 'Fig. 2 middle's. Since the terminal stretches had the same position land thus velocity and acceleration' profile, subtracting the two force responses cancelled any common static and dynamic terms, including those arising from passive tissues, inertial, and gravitational forces. The resulting response was approximately linear. The slope of this linear response with respect to position was interpreted as the active stiffness arising after reaching with respect to slow passive movement. To quantify the slope, regressions were performed on data drawn from each arm of each subject on a trial-bytrial basis [Fig. 2 middle'. These regressions were limited to the first $200 \mathrm{~ms}$ after the onset of the terminal stretch in order to minimize the possibility of voluntary intervention. The estimated slope from the linear regression ‘ $\mathrm{N} / \mathrm{cm}$ ' was defined as the terminal stiffness and this parameter was used in subsequent statistical analyses.

\section{RESULTS}

We measured reaching movements and mechanical properties of both arms of five spastic hemiparetic subjects using the ARM Guide IFig. 1'J. Movement amplitudes and velocities for spastic arms are summarized in Table 1. As was previously observed, ${ }^{30}$ all subjects showed repeatable active range of motion with the spastic arm as they reached along the device. The standard deviation of the final hand resting position was less than $1.5 \mathrm{~cm}$ for all subjects, while mean movement amplitudes ranged from 7.0 to $16.0 \mathrm{~cm}$ across subjects. For all subjects, the well-defined limit to active range of motion occurred at least $7.0 \mathrm{~cm}$ before the end of the passive range of motion of the arm determined manually by the experimenter.

All subjects developed large, perpendicular forces against the ARM Guide with the spastic arm during reaching. The forces were greatest in the horizontal plane, being medially directed li.e., in the positive $x$ direction, Fig. 1', and reached a maximum near the end of the range of motion 'Fig. 2 bottom'. The horizontal contact force at the end of the range of motion was significantly more medial by more than $20.0 \mathrm{~N}$ than the horizontal force generated by the contralateral arm for all subjects 'one-sided t-test, $\boldsymbol{p}=0.0001$ '. The increased horizontal contact force could not be attributed to passive properties of the spastic arm 'Fig. 2 bottom'. We have shown previously that these gross patterns of abnormal coordination are consistent with clinical descriptions of the abnormal extension muscle synergy. ${ }^{30}$

\section{Assessment of Passive Tissue Restraint}

To assess the level of passive tissue restraint at the workspace boundary, the ARM Guide returned the subject's hand to the initial starting position from which the most recent reach was initiated and slowly moved the relaxed arm back to the workspace boundary IFig. 2 top'. During these slow passive movements, EMG recordings of seven muscles surrounding the shoulder and elbow verified that muscles were inactive le.g., Fig. 5'.

For four of five subjects, the passive restraint force of the spastic arm measured at the workspace boundary was significantly greater than the restraint force generated by the contralateral arm after a matched stretch IFig. 3 top, t-test, $\boldsymbol{p}=0.05$ '. The average increase in restraint force across all subjects was $4.6 \mathrm{~N}$ 'SD $0.8 \mathrm{l}$. The increases in restraint force were decomposed in terms of shoulder and elbow torques using the two-joint model of the arm described in the Methods section. All subjects showed significant increases in elbow restraint torque, while four subjects showed significant increase in shoulder restraint torque ‘ $p=0.05$, Fig. 3 bottom'.

\section{Assessment of Active Muscle Restraint}

We hypothesized that any active restraint arising from activation of antagonist muscles during reaching would manifest itself as an increased stiffness following reaching, while the subject was still activating muscles and trying to move beyond the workspace boundary. To quantify active arm stiffness at the end of its active range 

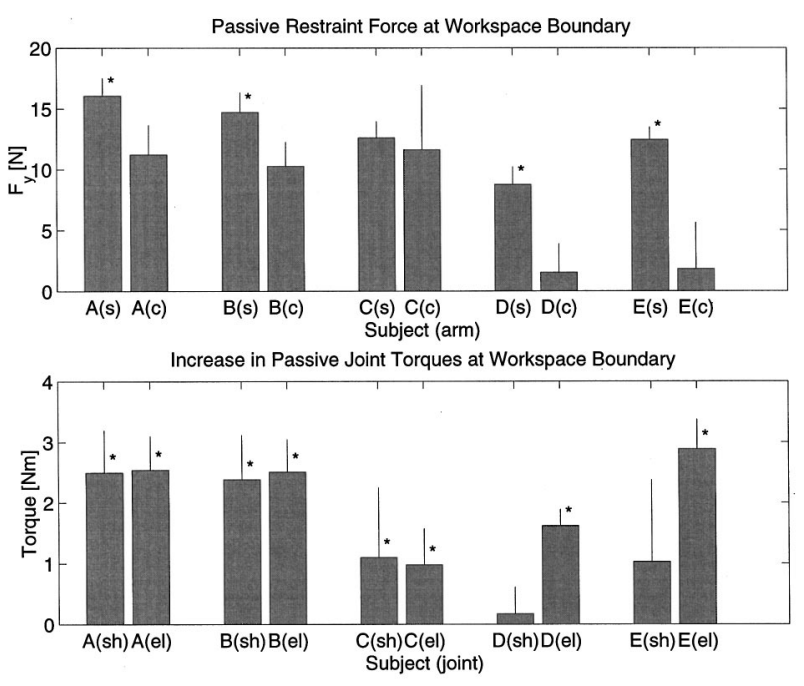

FIGURE 3. Top: Passive restraint force at the workspace boundary is: spastic arm; c: contralateral arm for subjects A-E!. Bars show one standard deviation. Asterisk indicates that restraint force generated by spastic arm was significantly greater than that generated by contralateral arm ittest, pr 0.05j. Bottom: Increase in passive joint restraint at the workspace boundary, estimated by inferring shoulder and elbow joint torques for each arm, and subtracting the contralateral torques from the spastic torques ish: shoulder joint; el: elbow joint j. Bars show one standard deviation. Asterisk indicates that the increase in the passive joint torque was significantly greater than zero it-test, $p=0.05$ ).

of motion, we applied a terminal stretch $150 \mathrm{~ms}$ following reaching, and compared it to the terminal stretch following slow passive movement through the same range. For all subjects, the difference between the restraint force in the two conditions, plotted as a function of hand position, was well approximated by a linear relationship [Fig. 2 middle'. The mean variance accounted for by linear regression of this relationship across all subjects was 0.86 [SD 0.05 for the spastic arms, and 0.85 ‘'SD 0.10' for the contralateral arms. As judged by the slope of the differential force response, the stiffness of the impaired arm following reaching was increased by an average of $5.3 \mathrm{~N} / \mathrm{cm}$ [SD 2.3' across subjects compared to arm stiffness following passive movement [Fig. 4’. Similarly, arm stiffness increased in the contralateral arm following matched reaching movements as compared to following passive movement by an average of $5.5 \mathrm{~N} / \mathrm{cm}$ ‘SD 1.6 '. These differences were significantly different from zero it-test, $p=0.001$ '], but not from each other. On a subject-by-subject basis, only subject $\mathrm{A}$ had a significantly greater active stiffness in the spastic arm It-test, $\boldsymbol{p}=0.05 \mathrm{~J}$, while subject B had a significantly lower active stiffness in the spastic arm It-test, $\boldsymbol{p}=0.01 \mathrm{j}$.

Stretch reflex responses to terminal stretch were seen in EMG recordings in the spastic arm of one subject lin

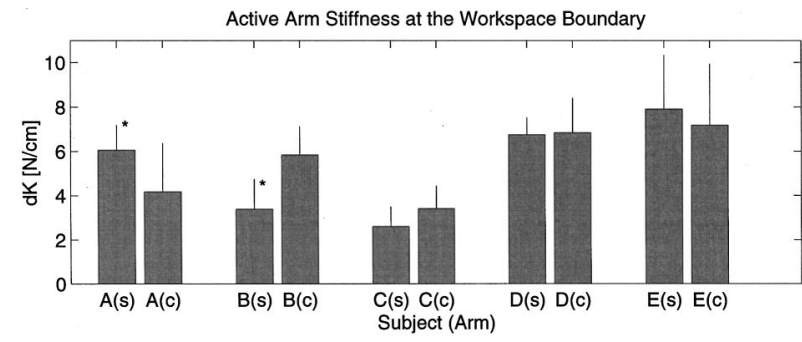

FIGURE 4. Active arm stiffness $\lceil d K$ at the workspace boundary is: spastic arm; c: contralateral arm for subjects A-E'. Bars show one standard deviation. Active arm stiffness was significantly greater than zero for all arms ‘t-test, pi 0.001 j. Asterisk indicates that spastic arm active stiffness was significantly different than that of the contralateral arm \{t-test, p-i 0.05].

brachioradialis following passive movement by subject B', raising the possibility that the terminal stiffness measurements were influenced by reflex activation of the arm due to the terminal stretch itself in this subject. The latency of the reflex response from the initiation of the terminal stretch for this subject was approximately 120 $\mathrm{ms}$, so, as a check, regressions were performed over only the first $120 \mathrm{~ms}$ after terminal stretch. The active stiffness for these regressions was not statistically different from those conducted over the first $200 \mathrm{~ms}$.

\section{EMG Patterns during Reaching}

Of interest with respect to the role of active restraint was the EMG behavior of antagonist muscles during reaching. Figure 5 shows ensemble-averaged EMG recordings from seven muscles of subject A from both the spastic and contralateral arms. These recordings exemplify several features of muscle activation that were exhibited across subjects. Reaching was initiated in both spastic and contralateral arms by activating the triceps and anterior deltoid muscles. In all spastic arms, pectoralis major was activated at the beginning of reaching, likely accounting at least in part for the large medial contact force generated against the ARM Guide. In both spastic and contralateral arms, activity was initiated in brachioradialis, posterior deltoid, and biceps at or soon after the beginning of movement, and was typically sustained in these antagonist muscles at levels above resting levels at the workspace boundary le.g., Fig. 5 'J. In the spastic arms, significant increases in the root-meansquare EMG amplitude in the $150 \mathrm{~ms}$ following the cessation of reaching compared with resting noise levels were found in either brachioradialis and biceps for all five subjects it-test, $\boldsymbol{p}=0.01 \mathrm{j}$, and in posterior deltoid in subjects $\mathrm{E}$ and $\mathrm{F}$ It-test, $\boldsymbol{p}=0.001 \mathrm{j}$. In the contralateral arms, significant increases were found in either brachio- 

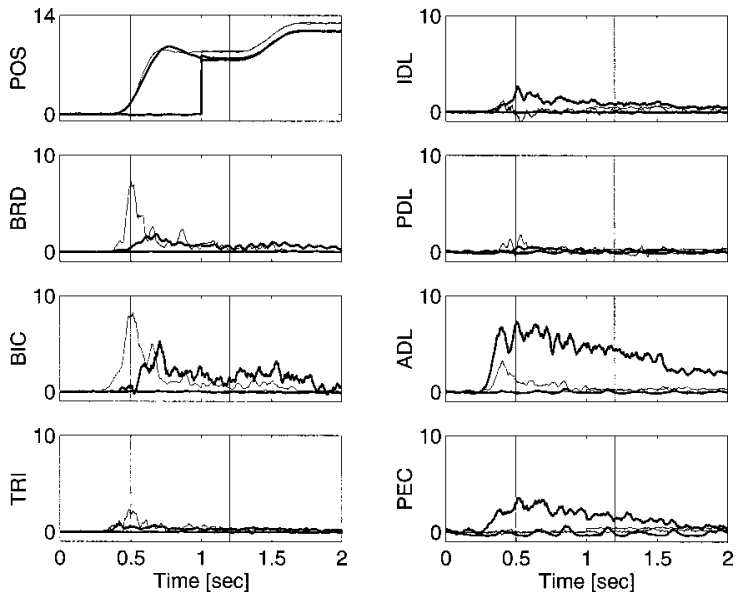

FIGURE 5. Example of EMG activity for subject $A$ during active reach and during passive restraint test for spastic arm (thick lines) and contralateral arm ‘thin linesi. Traces were formed by concatenating two ensemble-averaged traces. The first trace, from 0 to $1 \mathrm{~s}$, is the ensemble average of EMG recordings aligned at the time at which velocity crossed a small positive threshold. The second trace, from 1 to $2 \mathrm{~s}$, is the ensemble average of EMG recordings aligned at the time at which the terminal stretch began. Vertical lines denote alignment points. Position is measured in cm, EMG in arbitrary units, but equally scaled for different muscles. Note that only the beginning and end of the passive restraint test are shown iaccounting for discontinuity in position tracei. Note also that muscles remained inactive during the passive restraint test for both arms, except for a small amount of rhythmic activity in pectoralis, likely due to cross talk form heart electrical activity.

radialis and posterior deltoid for all five subjects lt-test, $\boldsymbol{p}=0.05 \mathrm{~J}$, and in biceps in three subjects it-test, $\boldsymbol{p}$ $<0.05$

\section{Assessment of Movement-Generated Weakness}

An additional experiment was performed to evaluate movement-generated weakness, defined as a decrease in force generating ability due to a preceding voluntary movement. In particular, a virtual "wall" was created using the motor to support the hand at the workspace boundary achieved by the previous reach, but allowing movement beyond the boundary. The wall thus allowed the subjects to continue reaching from the workspace boundary without a previous voluntary movement. EMG recordings confirmed that the muscles were inactive as the subjects rested against the virtual wall. When subjects tried to move beyond the virtual wall from this initially relaxed state, they could move farther [Fig. 6'. The mean increase in range across subjects was $2.6 \mathrm{~cm}$ [SD 0.6', with a mean peak velocity across subjects of $11.1 \mathrm{~cm} / \mathrm{s}$ 'SD 6.9'. The increased range for each subject, and the group average, were statistically greater than zero It-test, $p<=0.005$ '.

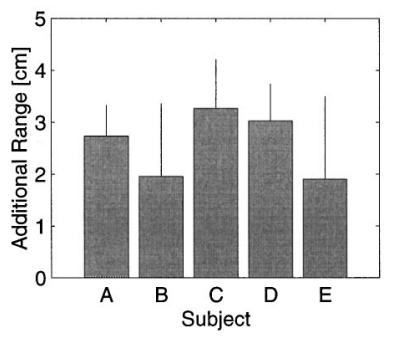

FIGURE 6. Enhanced range of motion when initiating movement from the workspace boundary. Bars show one standard deviation. Mean additional range was significantly greater than zero for all subjects ‘t-test, $p=0.05$ ! .

\section{DISCUSSION AND CONCLUSION}

These results demonstrate the use of a mechatronic device to assess functional movement after chronic brain injury. By allowing quantitative assessment of what is typically evaluated qualitatively, the device could help direct treatment planning. For example, all of the subjects exhibited increased passive tissue restraint at the elbow or shoulder, most likely resulting from disuse of the spastic arm. Treatment might thus be directed for these subjects toward passive range of motion exercise, which has been shown effective in addressing passive tissue restraint. ${ }^{\mathbf{1 5}}$ The effectiveness of the passive range of motion exercise could in turn be monitored using the ARM Guide.

Changes in passive tissue properties have been frequently observed in the lower extremity ${ }^{9,19,31,32}$ and have been suggested to occur at the elbow based on reduced EMG/torque ratios during rapid stretches..$^{\mathbb{1 0 , 2 0}}$ These changes likely occur from disuse of the spastic arm. ${ }^{15}$ However, a recent study from our laboratory found no significant increases in passive tissue properties at the elbow in a group of chronic stroke subjects. ${ }^{14}$ One possible explanation for the discrepancy of this previous result with the present study is that in the previous study subjects' elbows were held in an extended position for a prolonged time period between measurements. Such prolonged stretch may have caused a reduction in passive stiffness of the elbow, due to stress relaxation in muscle or soft tissues surrounding the joint, ${ }^{22}$ sufficient to mask increases in passive restraint force. Consistent with this explanation, we found significant shifts in force-length relationships of the arm after only several minutes of stretch in a case study of prolonged stretch using the ARM Guide. ${ }^{28}$

An unexpected result was that in four of five subjects the active stiffness of the spastic arms was not significantly greater than that of the contralateral arms. This result was surprising because all subjects had clinically detectable spasticity in their elbow flexor muscles, and exhibited signs of abnormal muscle coactivation, mani- 
fested in pectoralis major EMG recordings, and as large horizontal contact forces during reaching. Despite these potential indicators of active restraint, however, the stiffness measurements demonstrated that the net effect of reflex-based antagonist activation and abnormal antagonist coactivation was comparable to antagonist levels during reaching with the contralateral, nonparetic arm. This result is consistent with previous EMG studies suggesting that antagonist cocontraction is not excessive during either isometric force generation ${ }^{\mathbf{1} 1}$ or voluntary movement ${ }^{12}$ at the elbow in spastic hemiparesis.

The low levels of antagonist EMG activity that were present at the workspace boundary in the hemiplegic arms may have arisen from reflex activation of spastic muscles as they were stretched during reaching. Such reflex activation is one possible source of movementgenerated weakness, defined as a decrease in force generating ability due to a preceding voluntary movement. In our test for movement-generated weakness, the subjects were allowed to initiate reaching again from the workspace boundary, after allowing their arms to return to a relaxed state. Under these conditions, the subjects indeed were able to move $2-3 \mathrm{~cm}$ farther. One possible explanation is that the subjects simply fatigued during the full reaching movement, and were able to reach farther when given a change to rest. However, all subjects were capable of maintaining their arm at the workspace boundary for at least $150 \mathrm{~ms}$ following the full reaching movement, a result inconsistent with the presence of ongoing fatigue. A simpler hypothesis is that movementgenerated weakness reflects the presence of low-levels of persisting stretch reflex activation following reaching. In support of this hypothesis, preactivated, spastic elbow flexor muscles have been shown to exhibit a burst of activity when stretched, but then maintain a lower level of activity which persists for several seconds following stretch. $^{20,25}$

In conclusion, although the sample size was small, the results of this study suggest that a rational first approach to treating workspace deficits in chronic brain injury is to target passive tissue restraint and agonist weakness. Although we found evidence of active restraint at the workspace boundary, the restraint was typically comparable to that during reaching with the contralateral, nonparetic arm. We note that active restraint may play a greater role in some brain-injured patients, especially in limiting movement velocity or distorting movement trajectories. ${ }^{4}$ Additionally, measurements in this study were made for only one reaching direction, and the contributions of active and passive restraint may vary depending on the workspace area that is examined. Ultimately, mechatronic assessment techniques such as those developed here could provide a means to address problematic workspace areas on an individualized basis.

\section{ACKNOWLEDGEMENTS}

The authors gratefully acknowledge support of a Whitaker Foundation Biomedical Engineering Research Grant to DJR and NIDRR Field-Initiated Grant H133G80052.

\section{REFERENCES}

'Aisen, M. L., H. I. Krebs, N. Hogan, F. McDowell, and B. T. Volpe. The effect of robot-assisted therapy and rehabilitative training on motor recovery following stroke. Archives of Neurology 54:443-445, 1997.

${ }^{2}$ Ashworth, R. W. Preliminary trial of carisoprodaol in multiple sclerosis. Practitioner 192:540-542, 1964.

${ }^{3}$ Billian, C., and G. I. Zahalak. A programmable limb testing system land some measurements of intrinsic muscular and reflex-mediated stiffnessesi. J. Biomech. Eng. 105:6-11, 1983.

${ }^{4}$ Corcos, D. M., G. L. Gottlieb, R. D. Penn, B. Myklebust, and G. C. Agarwal. Movement deficits caused by hyperexcitable stretch reflexes in spastic humans. Brain 109:1043-1058, 1986.

${ }^{5}$ Craig, J. J. Introduction to Robotics: Mechanics and Control, 2nd ed. New York: Addison-Wesley, 1989.

${ }^{6}$ Delagi E. F., and A. Perotto. Anatomic Guide for the Electromyographer, 2nd ed. Springfield, IL: C. C. Thomas, 1980.

${ }^{7}$ Desrosiers, J., D. Bourbonnais, G. Bravo, P. M. Roy, and M. Guay. Performance of the 'unaffected' upper extremity of elderly stroke patients. Stroke 27:1564-1570, 1996.

${ }^{8}$ Dewald, J. P. A., P. S. Pope, J. D. Given, T. S. Buchanan, and W. Z. Rymer. Abnormal muscle coactivation patterns during isometric torque generation at the elbow and shoulder in hemiparetic subjects. Brain 118:495-510, 1995.

${ }^{9}$ Dietz, V., J. Quintern, and W. Berger. Electrophysiological studies of gait in spasticity and rigidity. Brain 104:431-449, 1981.

${ }^{10}$ Dietz, V., M. Trippel, and W. Berger. Reflex activity and muscle tone during elbow movements in patients with spastic paresis. Ann. Neurol. 30[6]:767-779, 1991.

${ }^{11}$ Fellows, S. J., C. Kaus, H. F. Ross, and A. F. Thilmann. Agonist and antagonist EMG activation during isometric torque development at the elbow in spastic hemiparesis. Electroencephalogr. Clin. Neurophysiol. 93:106-12, 1994.

${ }^{12}$ Fellows, S. J., C. Kaus, A. F. Thilmann. Voluntary movement at the elbow in spastic hemiparesis. Ann. Neurol. 36; 3]:397-407, 1994.

${ }^{13}$ Fugl-Meyer, A. R., L. Jaasco, L. Leyman, S. Olsson, and S. Steglind. The poststroke hemiplegic patient. Scand. J. Rehabil. Med. 7:13-31, 1975.

${ }^{14}$ Given, J. D., J. P. A. Dewald, and W. Z. Rymer. Joint dependent passive stiffness in paretic and contalateral limbs of spastic patients with hemiparetic stroke. J. Neurol., Neurosurg. Psychiatry 59:271-279, 1995.

${ }^{15}$ Goldspink, G., and P. E. Williams. Muscle fibre and connective tissue changes associated with use and disuse. In: Foundations for Practice: Topics in Neurological Physiotherapy, edited by L. Ada and C. Canning. London: Heinemann, 1990 pp. 197-218.

${ }^{16}$ Gomi, H., and R. Osu. Task-dependent viscoelasticity of human multijoint arm and its spatial characteristics for interaction with environments. J. Neurosci. 18\{21 \:8965-8978, 1998. 
${ }^{17}$ Gresham G. E., P. W. Duncan, and W. B. Stason. PostStroke Rehabilitation. Rockville, MD: U.S. Dept. Health and Human Services, Agency for Health Care Policy and Research; 1995.

${ }^{18}$ Hammond, M. C., S. S. Fitts, G. H. Kraft, P. B. Nutter, M. J. Trotter, and L. M. Robinson. Co-contraction in the hemiparetic forearm: quantitative EMG evaluation. Arch. Phys. Med. Rehabil. 69:348-51, 1988.

${ }^{19}$ Hufschmidt, A., and K.-H. Mauritz. Chronic transformation of muscle in spasticity: a peripheral contribution to increased tone. J. Neurol., Neurosurg. Psychiatry 48:676-685, 1985.

${ }^{20}$ Lee, W. A., A. Boughton, and W. Z. Rymer. Absence of stretch reflex gain enhancement in voluntarily activated spastic muscle. Exp. Neurol. 98:317-335, 1987.

${ }^{21}$ Lum, P. S., C. G. Burgar, D. Kenney, and H. F. M. Van der Loos. Quantification of force abnormalities during passive and active-assisted upper-limb reaching movements in poststroke hemiparesis. IEEE Trans. Biomed. Eng. 46 60;:652662, 1999.

${ }^{22}$ Magnusson, S. P. Passive properties of human skeletal muscle during stretch maneuvers. A review. Scand. J. Med. Sci. Sports 8[2]:65-77, 1998.

${ }^{23}$ Morgan, D. L. Separation of active and passive components of short-range stiffness of muscle. Am. J. Physiol. 232 1):C45-9, 1977.

${ }^{24}$ O'Dwyer, N. J., L. Ada, and P. D. Neilson. Spasticity and muscle contracture following stroke. Brain 119:1737-1749, 1996.

${ }^{25}$ Powers, R. K., D. L. Campbell, and W. Z. Rymer. Stretch reflex dynamics in spastic elbow flexor muscles. Ann. Neurol. 25:32-42, 1989.

${ }^{26}$ Reinkensmeyer, D. J., J. P. A. Dewald, and W. Z. Rymer. Robotic devices for physical rehabilitation of stroke patients: Fundamental requirements, target therapeutic techniques, and preliminary designs. Technology and Disability 5:205-215, 1996.

${ }^{27}$ Reinkensmeyer, D. J., N. Hogan, H. I. Krebs, S. L. Lehman, and P. S. Lum. Rehabilitators, robots, and guides: New tools for neurological rehabilitation, Biomechanics and Neural Control of Movement, edited by Jack Winters and Pat Crago New York: Springer, 1999, in press.

${ }^{28}$ Reinkensmeyer, D. J., and W. Z. Rymer. Using a mechanical suide to evaluate and treat workspace deficits after brain injury. Proceedings of the 19th Annual IEEE Engineering in Medicine and Biology Sociey Conference 1997.

${ }^{29}$ Reinkensmeyer, D. J., B. D. Schmit, and W. Z. Rymer. Arm stiffness at the active workspace boundary in spastic hemiparesis. Soc. Neurosci. Abstracts 24:1664, 1998.

${ }^{30}$ Reinkensmeyer, D. J., J. P. A. Dewald, and W. Z. Rymer. Guidance based quantification of arm impairment following brain injury: A pilot study. IEEE Trans. Rehabil. Eng. 7[1]:1-11, 1999.

${ }^{31}$ Sinkjaer, T., and I. Magnussen. Passive, intrinsic, and reflexmediated stiffness in the ankle extensors of hemiparetic patients. Brain 117:355-363, 1994.

${ }^{32}$ Thilmann, A. F., S. J. Fellows, H. F. Ross. Biomechanical changes at the ankle after stroke. J. Neurol., Neurosurg. Psychiatry 54:134-9, 1991. 